\title{
Altered expression of Aquaporin-2 in one-kidney, one-clip hypertension
}

\author{
Maria Florencia Albertoni Borghese ${ }^{\mathrm{a}, \mathrm{f}}$, Sandra Hope ${ }^{\mathrm{b}, \mathrm{c}}$, Maria del Carmen Ortiz ${ }^{\mathrm{a}}$, Magalí Barchuk ${ }^{\mathrm{a}}$, \\ Camila Kessler $^{\mathrm{a}}$, Carlos Davio ${ }^{\mathrm{d}, \mathrm{e}}$, Marcelo Vatta ${ }^{\mathrm{b}, \mathrm{c}}$, Mónica Majowicz ${ }^{\mathrm{a}, *}$ \\ ${ }^{a}$ Universidad de Buenos Aires, Facultad de Farmacia y Bioquímica, Departamento de Ciencias Biológicas, Cátedra de Biología Celular y Molecular, Buenos Aires, Argentina \\ ${ }^{\mathrm{b}}$ Universidad de Buenos Aires, Facultad de Farmacia y Bioquímica, Departamento de Ciencias Biológicas, Cátedra de Fisiología, Buenos Aires, Argentina \\ ${ }^{\mathrm{c}}$ Universidad de Buenos Aires, CONICET, Facultad de Farmacia y Bioquímica, Instituto de Química y Metabolismo del Fármaco (IQUIMEFA), Buenos Aires, Argentina

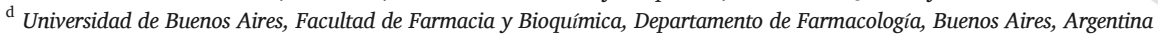 \\ e Universidad de Buenos Aires, CONICET, Facultad de Farmacia y Bioquímica, Instituto de Investigaciones Farmacológicas (ININFA), Buenos Aires, Argentina \\ ${ }^{\mathrm{f}}$ Universidad de Buenos Aires, CONICET, Facultad de Farmacia y Bioquímica, Departamento de Ciencias Biológicas, Cátedra de Biología Celular y Molecular, Buenos Aires, Argentina
}

\section{ART ICLE INFO}

\section{Keywords:}

Renovascular hypertension

Goldblatt 1-kidney

1-clip

Aquaporin-2

NFkB

Vasopressin V2 receptor

\begin{abstract}
A B S T R A C T
Aims: The aim of the present study was to evaluate the regulation of Aquaporin-2 (AQP2) water channel in the kidney of one-kidney, one-clip rats (Goldblatt-1 model). In addition, some mechanisms that underlie the role of AQP2 in the Goldblatt-1 model were evaluated.

Main methods: Sprague-Dawley rats were divided in three groups: control two-kidney, no clip (C, 2K-NC); nephrectomized one-kidney, no clip (N, 1 K-NC) and Goldblatt one-kidney, one-clip (G, 1 K-1C). AQP2 expression (by westernblot, real time PCR, immunohistochemistry and immunofluorescence), vasopressin V2 receptor expression (by real time PCR), cAMP concentration, NFkB and TonEBP (cytosol to nucleus ratio) were evaluated in the renal medulla.

Key findings: AQP2 expression, V2 receptor expression and cAMP concentration were decreased in the renal medulla of $1 \mathrm{~K}-1 \mathrm{C}$ rats, NFkB translocation was favoured towards the nucleus suggesting its activation while TonEBP translocation was not altered in this model of hypertension.

Significance: In this model of hypertension the decrease of AQP2 expression could be a mechanism that counteracts the high blood pressure promoting water excretion and this may be consequence of decreased vasopressin sensitivity and/or the increased activity of $\mathrm{NFkB}$ at renomedullary collecting duct level. Given that renovascular hypertension is among the most common causes of secondary hypertension, it is important to elucidate all the relevant mechanisms involved in the generation or in the compensation of the hypertensive state in order to improve the diagnoses and treatment of the patients.
\end{abstract}

\section{Introduction}

The kidney plays a central role in the regulation of salt and water balance, contributing to the control of arterial blood pressure. This regulation is achieved through the activity of both sodium and water transporters. This work has a special focus on water transport mediated by the aquaporins and in particular aquaporin-2 (AQP2). AQP2 plays a central role in the final regulation of water reabsorption in the renal collecting duct, being vasopressin (AVP) the main regulator of this process [1]. AVP exerts its effects acting through three different receptors: V1a, V1b (or V3) and V2 [2]. V1b receptors are present at central nervous system level, being involved in cognitive functions in the brain. Both V1a and V2 receptors are present in the kidney. V1a is associated with the vasa recta and is probably present in the intercalated cells of the cortical collecting duct. V2 receptor, the unique AVP receptor that signals through cAMP, has the key function of controlling fluid homeostasis [3]. Binding of AVP to V2 receptors in the basolateral membrane of principal cells in the collecting ducts stimulates adenylate cyclase producing cAMP, whose effects are mainly mediated by protein kinase A (PKA) [4, 5].

AVP regulates water permeability in the renal collecting duct by both short and long-term mechanisms. Short-term regulation occurs as a result of the modulation of trafficking of AQP2-containing membrane

\footnotetext{
* Corresponding author at: Cátedra de Biología Celular y Molecular, Facultad de Farmacia y Bioquímica, Universidad de Buenos Aires, Piso 1 (C1113AAD), Junín 956, Argentina. Email address: mmajow@ffyb.uba.ar (M. Majowicz)
} 
vesicles to and from the apical plasma membrane and long-term regulation occurs as a result of AQP2 expression regulation $[6,7]$.

Other proteins have been described as regulators of AQP2 expression, such as transcription factor tonicity responsive enhancer binding protein (TonEBP) [8] and the inflammatory mediator nuclear factor-kappaB (NFkB) [9]. TonEBP is a transcription factor activated under hypertonic conditions to protect renal cells from hypertonic stress stimulating the transcription of specific proteins [10]. Inflammation is a key feature in the initiation, progression and clinical implication of several cardiovascular diseases and the inflammatory response is essential in the pathogenesis of hypertension [11-13].

In this pathology, focus has been made on sodium transport but not on water transport, despite the importance of renal water handling in this pathological condition. There are no conclusive studies about the state of AQP2 during hypertension. However, some studies demonstrated changes in AQP2 targeting and expression in different experimental models of hypertension.

An increased expression and apical targeting of AQP2 in association with enhanced activity of the AVP/cAMP pathway has been reported in spontaneously hypertensive rats (SHR) [14-16]. On the other hand, a decrease in AQP2 expression was described in a Goldblatt-2 model of hypertension (two-kidney, one-clip; $2 \mathrm{~K}-1 \mathrm{C}$ ) [17, 18]. However, there are no reports on the status of AQP2 in the Goldblatt-1 model of hypertension (one-kidney, one-clip; $1 \mathrm{~K}-1 \mathrm{C}$ ). This renovascular model of hypertension described by Goldblatt is appropriate for studying the role of volume expansion in the development of hypertension $[19,20]$. Since in the model of $1 \mathrm{~K}-1 \mathrm{C}$ there is no other kidney, there is no pressure diuresis and natriuresis, there is rapid salt and water retention and hypertension soon becomes volume dependent and plasma renin activity usually remains normal after a short increase following the surgery [21, 22].

On these bases, the aim of the present study was to evaluate the regulation of AQP2 water channels in the kidney of one-kidney-one-clip rats. In addition, some mechanisms that underlie the role of AQP2 in the Goldblatt-1 model were evaluated.

\section{Materials and methods}

\subsection{Animals and hypertension model}

Male Sprague Dawley rats were purchased from Facultad de Ciencias Veterinarias -Universidad de Buenos Aires. Protocols were in compliance with ARRIVE guidelines, were designed according to the National Institutes of Health Guide for the Care and Use of Laboratory Animals, and were approved by the Institutional Committee for Use and Care of Laboratory Animals (CICUAL) from Facultad de Farmacia y Bioquímica. Forty-five-day-old rats weighing $150 \mathrm{~g}$ were anesthetized with Ketamine/Xylazine (50-10, $\mathrm{mg} / \mathrm{Kg}$ of body weight), the left kidney was exposed and a $0.25 \mathrm{~mm}$ gap silver clip was placed around the renal artery. For right nephrectomy, two ligatures were made around the renal vascular pedicle and the ureter. The kidney was then removed without the adrenal gland (Goldblatt rats). In another group of rats only the right kidney was extracted (nephrectomized rats) and a third group was subjected to a sham surgery procedure, with the exception of the artery clip and the nephrectomy (Control rats). All rats received $0.1 \mathrm{ml}$ $(40,000 \mathrm{IU})$ of amoxicillin at the end of the surgery.

Therefore we worked with three groups: control two-kidney-no clip (C, 2K-NC, $n=12$ ); nephrectomized one-kidney-no clip (N, $1 \mathrm{~K}-\mathrm{NC}$ $\mathrm{n}=12$ ) and Goldblatt hypertensive group one-kidney-one-clip ( $\mathrm{G}$, $1 \mathrm{~K}-1 \mathrm{C}, n=15$ ), which were studied four weeks after surgery.

\subsection{Determination of systolic blood pressure}

Systolic BP was recorded weekly in conscious rats by tail plethysmography (ADInstruments PowerLab 8/30 and NIBP Controller ML125).

\subsection{Determinations in the 24-h metabolic cage studies}

Animals were acclimatized to metabolic cages for 2 days before $24 \mathrm{~h}$ urine collection. Urine samples were analyzed for total protein concentration (Proti U/LCR Kit; Wiener Lab., Rosario, Argentina) and urine osmolality (OSMETTE, Precision Systems Inc., MA, USA). Urine volume was measured gravimetrically. Kinetic determinations of serum and urinary creatinine concentration were evaluated using a kit provided by Wiener. Urinary sodium was measured using an ion analyzer (Tecnolab; Mod. T-412, Argentina).

Blood collection was performed with the rats under urethane anesthesia ( $1 \mathrm{~g} / \mathrm{Kg}$ via i.p) by cardiac puncture; animals were killed by exsanguination, then both kidneys or the remaining kidney were immediately removed and weighed.

\subsection{Tissue processing for Western blot analysis in medulla homogenates}

Immediately after the animals were sacrificed, their kidneys were isolated and the renal medulla was dissected and homogenized in an appropriate homogenization buffer $(250 \mathrm{mmol} / 1$ sucrose, $1 \mathrm{mmol} / \mathrm{l}$ EDTA, $0.1 \mathrm{mmol} / 1 \mathrm{PMSF}$ and $10 \mathrm{mmol} / 1 \mathrm{Tris}-\mathrm{ClH}), \mathrm{pH} 7.6$. Large tissue debris and nuclear fragments were removed by a low-speed spin $(1000 \mathrm{~g}$, $10 \mathrm{~min}, 4^{\circ} \mathrm{C}$ ). Protein concentration was measured using BCA TM Protein Assay Kit (Pierce, Rockford, IL, USA).

\subsection{Western blot for AQP2}

Immunoblotting analysis was used to identify AQP2. AQP2 antibody (rabbit anti-rat AQP2; Santa Cruz Biotechnology, Inc., CA, USA) was followed by a donkey anti-rabbit IgG (HRP) secondary antibody (Abcam Inc., Cambridge, MA, USA) and revealed using Bio-Lumina kit (Kalium Technologies; Buenos Aires, Argentina). Beta-tubulin was used as loading control (Abcam). AQP2 antibody recognizes at least two bands of 29 and 35-40 kDa corresponding to unglycosylated and glycosylated AQP2, respectively.

The relative protein levels were analyzed with Gel Pro Analyzer 3.1 for Windows and the ratio of AQP2 to beta-tubulin was calculated.

\subsection{Tissue processing for Western blot analysis in cytosolic fraction and purified nuclei}

After homogenization of the renal medulla, the homogenate was centrifuged for $10 \mathrm{~min}$ at $800 \times g$ to obtain the nuclear fraction in the pellet and the cytosolic fraction in the supernatant. The pellet was washed with homogenization buffer and suspended in a solution of $2.4 \mathrm{M}$ sucrose followed by a centrifugation at $50000 \times g$ for $1 \mathrm{~h}$ to obtain the purified nuclei in the pellet.

\subsection{Western blots for NFkB and TonEBP}

Immunoblotting analysis was used to identify NFkB or TonEBP in the cytosolic fraction and in purified nuclei. The appropriate primary antibody (mouse anti-rat NFkB, Santa Cruz Biotechnology, or rabbit anti-rat TonEBP; Santa Cruz Biotechnology) was followed by a secondary antibody (Horse anti mouse HRP, GE Healthcare, UK or donkey anti-rabbit IgG HRP Abcam) and revealed as described above. NFkB 
antibody recognizes a $65-\mathrm{kDa}$ band corresponding to p65 subunit and TonEBP antibody recognizes a 170-KDa band.

Beta-tubulin was used as loading control for the cytosolic fraction and Lamina (Mouse anti rat Millipore MA, USA) was used as loading control for the purified nuclei. The relative protein levels were analyzed with Gel Pro Analyzer 3.1 for Windows and the ratio of cytosolic to nuclei expression was used as an indicator of transcription factor activation.

\subsection{Real-time PCR for AQP2 and V2 receptor}

Total RNA was isolated using the SV total RNA Isolation System (Promega) and reverse transcribed to cDNA using a high capacity reverse transcription kit (A\&B, MA, USA). For real-time detection of AQP2 and V2 transcripts and the reference gene (GAPDH), MezclaReal (Biodynamics, Buenos Aires, Argentina) and specific primers were used [23, 24]. The normalized gene expression method $\left(2^{-\Delta \Delta \mathrm{CT}}\right)$ for the relative quantification of gene expression was used [25].

\section{9. cAMP measurements}

cAMP was measured in the renal medulla. Approximately $100 \mathrm{mg}$ of renal medulla was homogenized in ice-cold absolute ethanol and centrifuged for $15 \mathrm{~min}$ at $1200 \mathrm{~g}$. The supernatant was dried, and the remaining residue was suspended for cAMP determination by competition of [3H]-cAMP for PKA [26]. Results were expressed as pmoles/mg of protein.

\subsection{Immunohistochemistry for $A Q P 2$}

Five micrometer slices were incubated with primary rabbit polyclonal antibody to AQP2 (Abcam) and a secondary biotinylated donkey anti rabbit (Jackson ImmunoResearch, West Grove, PA, USA), followed by the streptavidin-biotin-peroxidase reaction (Dako Cytomation, Glostrup, Denmark) visualized by exposure to diaminobenzidine (DAB) $-\mathrm{H}_{2} \mathrm{O}_{2}$. Endogenous peroxidase activity was quenched with hydrogen peroxide to prevent unspecific staining. Tissue sections were counterstained with hematoxylin.

\subsection{Immunofluorescence for $A Q P 2$}

Kidneys were fixed with $4 \%$ paraformaldehyde, pH7.4, cryoprotected in $5 \%$ and $25 \%$ sucrose/PBS, pH7.4 and frozen at $-70^{\circ} \mathrm{C}$. Then the kidneys were cut in $5 \mu \mathrm{m}$ slices. Kidney sections were incubated with a primary rabbit polyclonal antibody to AQP2 (Abcam) followed by a secondary FITC labeled donkey anti rabbit antibody (Alexa fluor 488, goat anti rabbit Ig G; Invitrogen) and mounted with Vectashield Mounting Medium (Vector Laboratories, Burlingame, CA, USA).

\subsection{Image capture and analysis}

All determinations were performed blindly and under similar light, gain and offset conditions by the same operator.

Images from immunohistochemical sections were captured using a Nikon Alphaphot-2 YS2 light microscope (Nikon Instrument Group, Melville, NY), coupled to a Sony color video camera digital (Model No. SSC-DC50A).

Images from immunofluorescence were examined by wide-field microscopy with an epifluorescence Nikon Eclipse Ti microscope. Images were taken with the acquisition freeware Micrometric SE premium.

\subsection{Statistics}

One-way ANOVA with Bonferroni's post-test for multiple comparisons was performed using Graph Pad Prism version 5.0 for Windows.

\section{Results \\ 3.1. Characterization of the animal model}

Data for renal functional parameters and systolic BP 4 weeks after the surgical procedure are shown in Table 1.

Systolic BP was significantly higher in $1 \mathrm{~K}-1 \mathrm{C}$ group compared to control and $1 \mathrm{~K}-\mathrm{NC}$ groups $(p<0.001)$. Kidney weight/100g b.w. increased in both $1 \mathrm{~K}-\mathrm{NC}(p<0.01)$ and $1 \mathrm{~K}-1 \mathrm{C}(\mathrm{p}<0.001)$ groups compared to the control group meanwhile there were no differences in body weight between the three groups. Kidney size increases in a number of physiological and pathological situations, including uninephrectomy. Renal mass increases after nephrectomy mainly in the cortex, particularly in the proximal convoluted tubules, and appears to be of a hypertrophic, rather than hyperplastic nature and is mediated by a cell cycle-dependent mechanism [27].

Urinary volume significantly increased in $1 \mathrm{~K}-1 \mathrm{C}$ group compared to control group $(p<0.05)$, meanwhile only had a tendency to increase in $1 \mathrm{~K}-\mathrm{NC}$ group. Urinary osmolality decreased in $1 \mathrm{~K}-1 \mathrm{C}$ group compared to both $1 \mathrm{~K}-\mathrm{NC}$ and the control group $(p<0.05)$.

Table 1

Systolic blood pressure and renal functional parameters.

\begin{tabular}{|c|c|c|c|}
\hline & $2 \mathrm{~K}-\mathrm{NC}$ & $1 \mathrm{~K}-\mathrm{NC}$ & $1 \mathrm{~K}-1 \mathrm{C}$ \\
\hline Systolic arterial pressure $(\mathrm{mm} \mathrm{Hg})$ & $124.7 \pm 0.9(n=7)$ & $123.6 \pm 1.0(\mathrm{n}=7)$ & $185.0 \pm 15.9 * * *, \# \# \#(n=7)$ \\
\hline Kidney weight (g) & $1.46 \pm 0.04(n=12)$ & $2.13 \pm 0.10^{* * *}(\mathrm{n}=12)$ & $2.26 \pm 0.13^{* * *}(n=15)$ \\
\hline Body weight (g) & $348.1 \pm 15.8(n=11)$ & $331.0 \pm 22.0(n=11)$ & $320.6 \pm 12.6(n=14)$ \\
\hline Kidney weight/100g b.w. (g/100g) & $0.35 \pm 0.03(\mathrm{n}=11)$ & $0.59 \pm 0.02^{* *}(\mathrm{n}=11)$ & $0.67 \pm 0.05^{* * *}(n=14)$ \\
\hline Urinary volume $(\mathrm{ml} / 24 \mathrm{~h})$ & $8.8 \pm 1.1(n=6)$ & $13.3 \pm 1.8(n=10)$ & $18.1 \pm 2.5 *(n=10)$ \\
\hline Urinary Osmolality (mOSm/L) & $1870 \pm 210(n=6)$ & $1910 \pm 169(n=6)$ & $964 \pm 245^{*, \#}(n=7)$ \\
\hline Urinary sodium (meq/24h) & $2.98 \pm 0.95(n=6)$ & $3.44 \pm 0.88(n=6)$ & $2.88 \pm 0.47(n=7)$ \\
\hline Proteinuria $(\mathrm{mg} / 24) \mathrm{h} / 100 \mathrm{~g})$ & $3.12 \pm 0.60(n=6)$ & $4.41 \pm 0.42(n=6)$ & $5.67 \pm 0.72 *(n=6)$ \\
\hline Creatinine clearance $(\mathrm{ml} / \mathrm{min} / 100 \mathrm{~g})$ & $0.37 \pm 0.07(n=6)$ & $0.32 \pm 0.02(n=6)$ & $0.37 \pm 0.10(n=6)$ \\
\hline
\end{tabular}

$2 \mathrm{~K}-\mathrm{NC}$ : control group; $1 \mathrm{~K}-\mathrm{NC}$ : one kidney-no clip; $1 \mathrm{~K}-1 \mathrm{C}$ : one kidney-1 clip hypertensive group. One-way ANOVA followed by Bonferroni's test for multiple comparisons.

$* \mathrm{p}<0.05$ vs $2 \mathrm{~K}-\mathrm{NC}$.

$* * \mathrm{p}<0.01$ vs $2 \mathrm{~K}-\mathrm{NC}$.

*** $p<0.001$ vs. $2 \mathrm{~K}-\mathrm{NC}$

\# $\mathrm{p}<0.05$ vs. $1 \mathrm{~K}-\mathrm{NC}$.

\#\#\# $\mathrm{p}<0.001$ vs. $1 \mathrm{~K}-\mathrm{NC}$. Data are mean \pm SEM. 
Proteinuria significantly increased in $1 \mathrm{~K}-1 \mathrm{C}$ group when compared to control group $(\mathrm{p}<0.05)$. There were no significant differences in creatinine clearance and urinary sodium excretion among the three groups.

Aquaporin-2 expression is decreased in the renal medulla of the hypertensive $1 \mathrm{~K}-1 \mathrm{C}$ rats.

Fig. 1 shows that both AQP2 protein and mRNA expression were decreased in the renal medulla of $1 \mathrm{~K}-1 \mathrm{C}$ rats compared to both $1 \mathrm{~K}-\mathrm{NC}$ and control groups $(p<0.05)$. We evaluated AQP2 expression both at protein and mRNA levels because uncoupled expression between AQP2 mRNA and protein has been described under certain conditions [28].

The results of AQP2 Western blot were corroborated by immunohistochemistry and immunofluorescence (Fig. 2). The images obtained by

A1

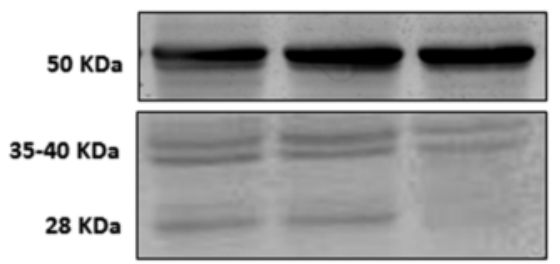

A2

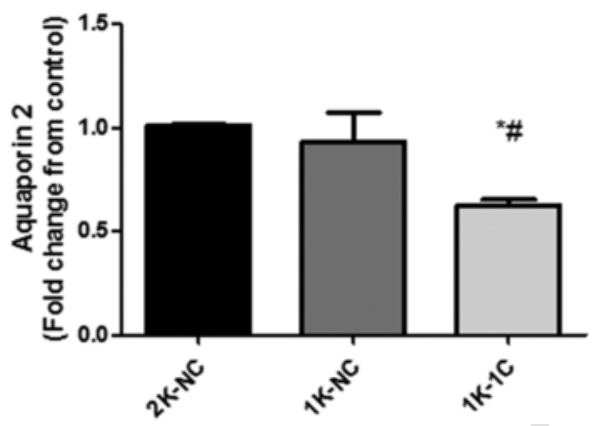

immunofluorescence show AQP2 labeling both in the cytosol and in the apical membranes of the principal cells for the three groups, there were no net changes in AQP2 translocation in none of the studied groups.

\subsection{V2 receptor expression and CAMP production are decreased in the renal medulla of hypertensive $1 \mathrm{~K}-1 \mathrm{C}$ rats}

V2 receptor mRNA expression decreased in the renal medulla of $1 \mathrm{~K}-1 \mathrm{C}$ rats compared to both $1 \mathrm{~K}-\mathrm{NC}$ and control groups $(p<0.01)$, as can be shown in Fig. 3A.

Fig. 3B shows that cAMP production decreased in the renal medulla of $1 \mathrm{~K}-1 \mathrm{C}$ rats compared to both $1 \mathrm{~K}-\mathrm{NC}$ and control groups $(\mathrm{p}<0.01)$.

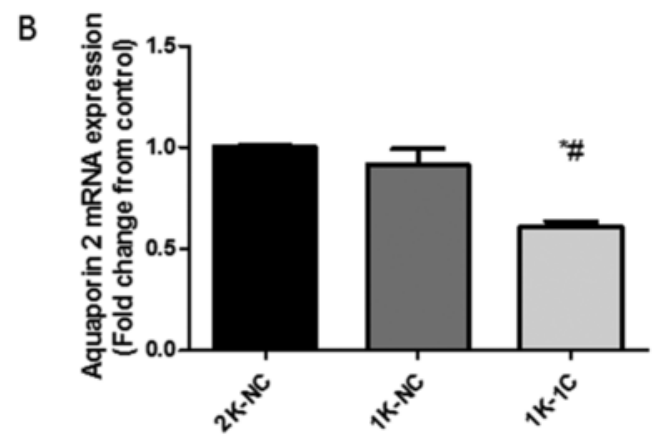

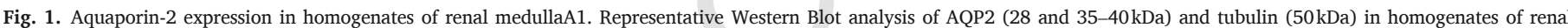

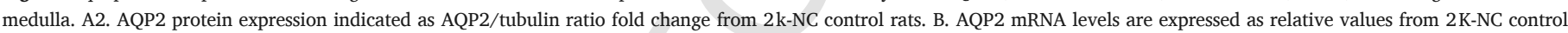

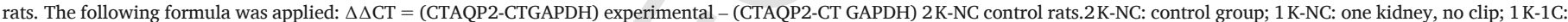

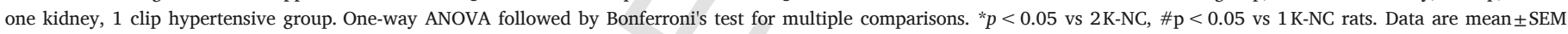
$(n=6)$.
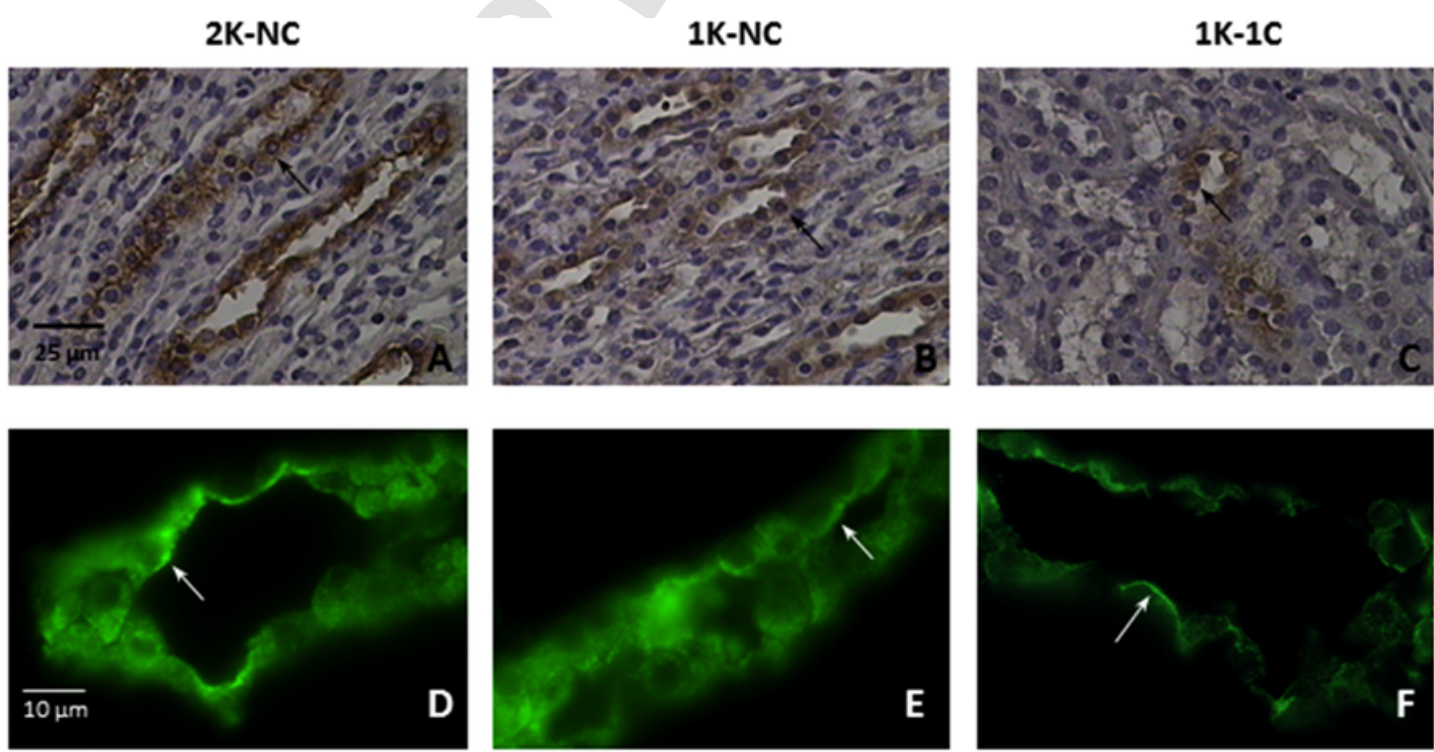

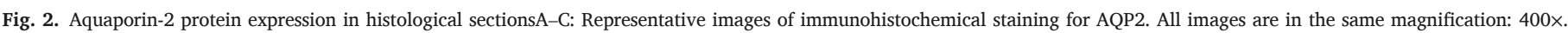

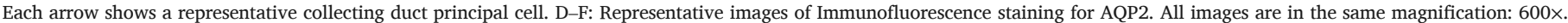
Each arrow shows apical staining for AQP2.2K-NC: control group; $1 \mathrm{~K}-\mathrm{NC}$ : one kidney, no clip; $1 \mathrm{~K}-1 \mathrm{C}$ : one kidney, 1 clip hypertensive group; ( $n=3$ ). 
A

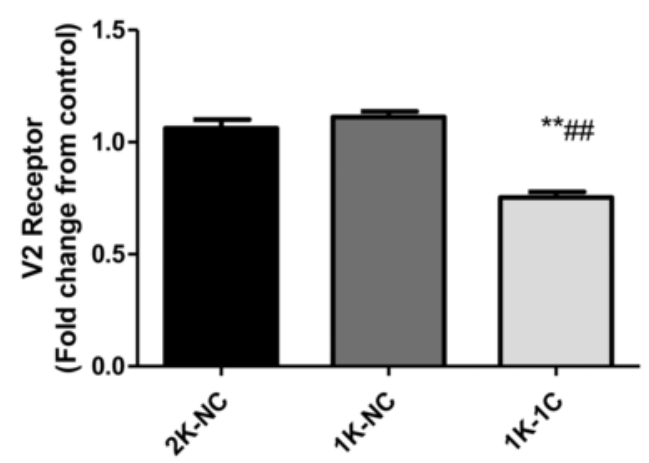

B

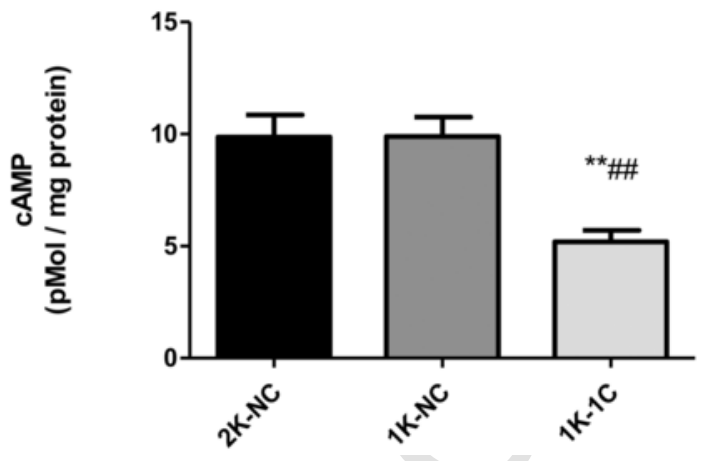

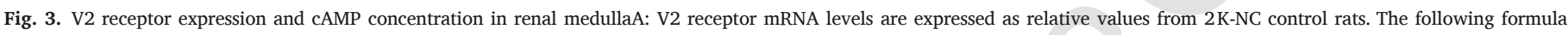

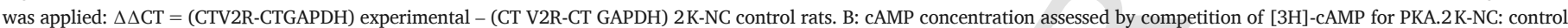

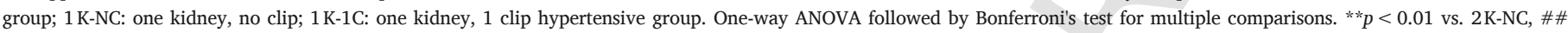
$\mathrm{p}<0.01$ vs. $1 \mathrm{~K}-\mathrm{NC}$ rats. Data are mean $\pm \operatorname{SEM}(n=4)$.

\section{3. $N F k B$ is activated in hypertensive $1 \mathrm{~K}-1 \mathrm{C}$ rats}

Fig. 4A shows that cytosolic/nuclear ratio of NFkB expression decreased in hypertensive $1 \mathrm{~K}-1 \mathrm{C}$ rats, due to the increase in NFkB nuclear localization. NFkB was shown to negatively regulate AQP2 expression, so this activation of NFkB could be contributing to AQP2 downregulation.

\subsection{TonEBP translocation is not altered in the renal medulla of hypertensive $1 \mathrm{~K}-1 \mathrm{C}$ rats}

Fig. 4B shows that cytosolic/nuclear ratio of TonEBP expression did not change in $1 \mathrm{~K}-1 \mathrm{C}$ animals when compared to control group or to the nephrectomized group, indicating that Ton EBP is not activated in this model of hypertension.
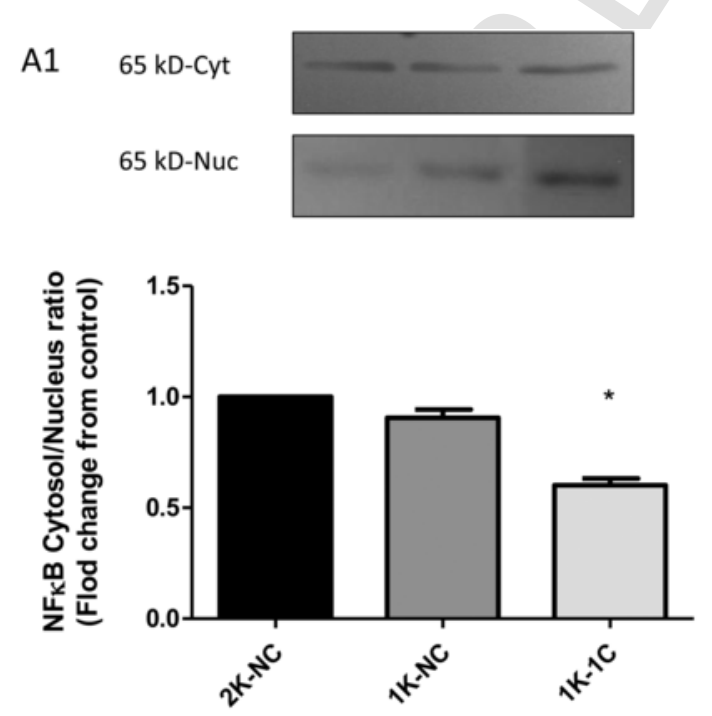

A2

\section{Discussion}

In this work we show that AQP2 expression is altered in $1 \mathrm{~K}-1 \mathrm{C}$ hypertension with a decrease in both protein expression and mRNA levels. The decrease in AQP2 expression results in an altered urinary concentration that leads to an increased urinary volume and a decreased urinary osmolality in $1 \mathrm{~K}-1 \mathrm{C}$ hypertensive group. The fact that AVP pathway is also altered may explain, at least in part, the diminished AQP2 expression and the increased diuresis found in this hypertension model. These results are in accordance with those reported by Lee et al., who also showed a reduced expression of AQP2 without changes in its translocation and a diminished cAMP production in the clipped kidney from $2 \mathrm{~K}-1 \mathrm{C}$ rats, another model of Goldblatt renovascular hypertension [17].

Contrary to results mentioned above, it was reported that the medullary expression and apical targeting of AQP2 and adenylate cyclase activity are increased in the kidney of SHR, contributing to the pathogenesis of hypertension $[14,16]$. Similarly, in the kidney of

B1

\section{0 kD-Cyt}

170 kD-Nuc
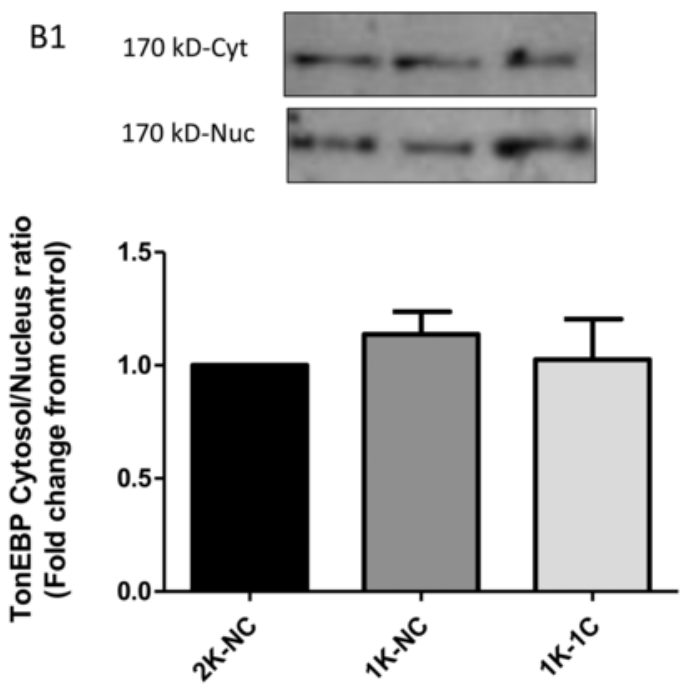

B2

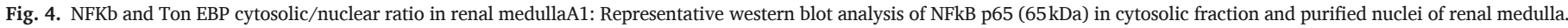

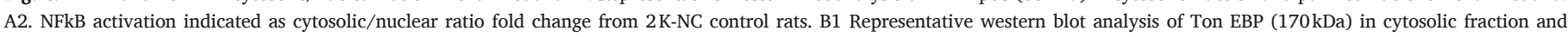

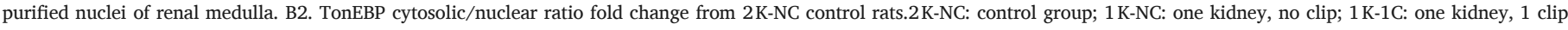
hypertensive group. One-way ANOVA followed by Bonferroni's test for multiple comparisons. ${ }^{*} p<0.05$ vs. 2 K-NC. Data are mean \pm SEM ( $n=3$ ). 
DOCA-salt hypertensive rats, a model where hypertension maintenance has been attributed to increased AVP, cAMP and AQP2 expression and shuttling are increased suggesting that they are causally related with the development of hypertension [29].

In this work we show a decrease in V2 receptor expression and cAMP reduction in the renal medulla of $1 \mathrm{~K}-1 \mathrm{C}$ rats. AVP plays an important role in the regulation of BP by means of $\mathrm{V} 1$ and $\mathrm{V} 2$ receptors; however the regulation of the expression of these receptors in hypertension is not completely understood. Burrell et al. demonstrated that in SHR, increase of plasma AVP and activation of renal V1a receptor occurs during developing hypertension, with downregulation when hypertension is established [24]. Contrary to our results, they did not observe changes in V2 receptor expression, but the study was conducted in a different model of hypertension $[17,30]$. Some authors have shown that plasma AVP is elevated in rats with $1 \mathrm{~K}-1 \mathrm{C}$ Goldblatt hypertension [31, 32]. Share et al. pointed out that AVP may contribute to $1 \mathrm{~K}-1 \mathrm{C}$ hypertension by virtue of its antidiuretic action, allowing the expansion of blood volume that apparently is necessary for the chronic stage of the hypertensive state [33]. Lariviere et al. showed that together with an increased concentration of AVP in plasma, the number of vascular AVP-binding sites is decreased in $1 \mathrm{~K}-1 \mathrm{C}$ hypertensive rats [32]. So it is possible that increased AVP levels leads to a decrease in renal medullary V2 receptor expression as a compensatory mechanism, diminishing AVP sensitivity and consequently decreasing cAMP levels and AQP2 expression in order to counteract the volume expansion.

On the other hand, the reduction in cAMP production and consequently AQP2 decrease may be consequence of the ischemic condition of the clipped kidney $[17,34]$, which in turn promotes oxidative stress. It is known that an increase in oxidative stress plays a role in renovascular hypertension $[35,36]$ and that the interaction between reactive oxygen species (ROS) and NO reduces its bioavailability [37] In a previous work we demonstrated that AQP2 is down-regulated in the renal medulla of rats made hypertensive by chronic inhibition of nitric oxide synthase [38] and later we provided evidence that NO has a role in the control of AQP2 expression [39]. Besides the decrease in NO bioavailability, the interaction of ROS with NO promotes the increase in pro-oxidant peroxynitrite. The combination of diminished NO bioavailability and increased peroxynitrite impairs intrarenal vascular, glomerular and tubular function [37].

TonEBP, a protein known to regulate AQP2 expression by enhancing its gene transcription independently of AVP action, is not activated in this model of hypertension, since this is a volume-dependent hypertension model and TonEBP is activated in hypertonic environments [40].

As expected, NFkB is activated in this model of hypertension which courses with the development of pro-inflammatory mechanisms. It was already shown that inflammatory processes contribute to the increased arterial pressure in human essential arterial hypertension and in different animal models of experimental hypertension [41]. Besides, it is known that NFkB activation is important for the downregulation of AQP2 channel and vasopressin V2 receptor expression during sepsis [42] and other inflammatory diseases [9]. Hasler et al. identified NFkB as a negative acting transcription factor that physiologically controls AQP2 transcription and may antagonize both CREB and TonEBP stimulatory effects. In that article the authors said that the strong activation of NFkB observed in renal inflammatory diseases is most likely to account for the observed decrease of AQP2 expression and accompanies urinary concentration defects [9]. The analysis of AQP2 promoter revealed two putative $\mathrm{kB}$ binding elements. NFkB stimulates or represses target gene transcription depending on the nature of the bound $\mathrm{NFkB}$ homo- or heterodimer and on bound NFkB cofactors [9]. The differential dimerization between NFkB family members allows for cell-specific transcriptional modulation of target genes in response to a great number of stimuli [9]. Hasler et al. showed that LPS treatment of cultured mouse CD principal cells or rat kidney slices decreased AQP2 expression, being this effect mediated by decreased p65 and increased p50 and p52 binding to kB elements of AQP2 promoter [9]. This result is opposite to our finding because we found that in $1 \mathrm{~K}-1 \mathrm{C}$ hypertensive Goldblatt model AQP2 expression decreases as a consequence of increased nuclear localization of p65. However, in accordance to us Lin et al. found that knockdown of NF-kBp65 or NF-kBp65 inhibition by pyrrolidine dithiocarbamate (PDTC) enhanced AQP2 expression in IMCD cells exposed to TNF- $\alpha$ [43].

\section{Conclusions}

Our results allow us to conclude that, in Goldblatt-1 hypertensive model, the decrease of AQP2 could be counterbalancing the enhancement in BP by increasing water excretion as a consequence of the decrease in vasopressin sensitivity, although this needs further investigation. On the other hand, we cannot discard activation of $\mathrm{NFkB}$ as a mechanism contributing to AQP2 downregulation although it would be hard to conceive inflammation as a compensatory mechanism for hypertension since it is actually one of the initiating factors.

Hypertension is one of the leading causes of morbidity and mortality and increases every year. Renovascular hypertension is among the most common causes of secondary hypertension [37]. Therefore, it is very important to elucidate all the relevant mechanisms involved in the generation or in the compensation of the hypertensive state in order to improve the diagnoses and treatment of the patients.

\section{Acknowledgements}

This work was supported by Grants UBACYT 20020130200184BA and 20020120300009BA from the Universidad de Buenos Aires to MPM and MFAB respectively. The funders had no role in study design, data collection and analysis, decision to publish, or preparation of the manuscript. We would like to thank Dr. Maria del Carmen Fernandez who kindly gave us TonEBP antibody.

\section{Conflict of interest}

The authors declare that there are no conflicts of interest.

\section{Contributions}

Conceived and designed the experiments: MM and MV. Performed the experiments: MFAB, SH, MB, MCO, CK, CD, MV and MM. Analyzed the data: MFAB, SH, MCO, MV and MM. Contributed reagents/materials/analysis tools: MV, CD, MFAB and MM. Wrote the paper: MFAB, MV and MM. Interpreted results of experiments: MFAB, SH, MV and MM. Prepared figures: MFAB and MM. Edited and revised the manuscript: MFAB, MCO, MV and MM.

\section{References}

[1] C. Esteva-Font, J. Ballarin, P. Fernandez-Llama, Molecular biology of water and salt regulation in the kidney, Cell. Mol. Life Sci. 69 (5) (2012 Mar) 683-695.

[2] S. Jard, Vasopressin receptors. A historical survey, Adv. Exp. Med. Biol. 449 (1998) $1-13$.

[3] K.V. Juul, D.G. Bichet, S. Nielsen, J.P. Norgaard, The physiological and pathophysiological functions of renal and extrarenal vasopressin V2 receptors, Am. J. Physiol. Ren. Physiol. 306 (9) (2014) F931-F940.

[4] H.B. Moeller, R.A. Fenton, Cell biology of vasopressin-regulated aquaporin-2 trafficking, Pflugers Arch. 464 (2) (2012 Aug) 133-144.

[5] P.I. Nedvetsky, G. Tamma, S. Beulshausen, G. Valenti, W. Rosenthal, E. Klussmann, Regulation of aquaporin-2 trafficking, Handb. Exp. Pharmacol. 190 (2009) 133-157.

[6] J.L. Wilson, C.A. Miranda, M.A. Knepper, Vasopressin and the regulation of aquaporin-2, Clin. Exp. Nephrol. 17 (6) (2013 Dec) 751-764.

[7] S. Nielsen, C.L. Chou, D. Marples, E.I. Christensen, B.K. Kishore, M.A. Knepper, Vasopressin increases water permeability of kidney collecting duct by inducing 
translocation of aquaporin-CD water channels to plasma membrane, Proc. Natl. Acad. Sci. U. S. A. 92 (4) (1995 Feb 14) 1013-1017.

[8] U. Hasler, U.S. Jeon, J.A. Kim, D. Mordasini, H.M. Kwon, E. Feraille, et al., Tonicity-responsive enhancer binding protein is an essential regulator of aquaporin-2 expression in renal collecting duct principal cells, J. Am. Soc. Nephrol. 17 (6) (2006 Jun) 1521-1531.

[9] U. Hasler, V. Leroy, U.S. Jeon, R. Bouley, M. Dimitrov, J.A. Kim, et al., NF-kappaB modulates aquaporin-2 transcription in renal collecting duct principal cells, J. Biol. Chem. 283 (42) (2008 Oct 17) 28095-28105.

[10] S.K. Woo, H.M. Kwon, Adaptation of kidney medulla to hypertonicity: role of the transcription factor TonEBP, Int. Rev. Cytol. 215 (2002) 189-202.

[11] D. Tsounis, G. Bouras, G. Giannopoulos, C. Papadimitriou, D. Alexopoulos, S. Deftereos, Inflammation markers in essential hypertension, Med. Chem. 10 (7) (2014) 672-681.

[12] L.E. Bautista, L.M. Vera, I.A. Arenas, G. Gamarra, Independent association between inflammatory markers (C-reactive protein, interleukin-6, and TNF-alpha) and essential hypertension, J. Hum. Hypertens. 19 (2) (2005 Feb) 149-154.

[13] B. Rodriguez-Iturbe, Y. Quiroz, A. Ferrebuz, G. Parra, N.D. Vaziri, Evolution of renal interstitial inflammation and NF-kappaB activation in spontaneously hypertensive rats, Am. J. Nephrol. 24 (6) (2004 Nov) 587-594.

[14] M. Buemi, L. Nostro, P.G. Di, E. Cavallaro, A. Sturiale, F. Floccari, et al., Aquaporin-2 water channels in spontaneously hypertensive rats, Am. J. Hypertens. 17 (12 Pt 1) (2004 Dec) 1170-1178.

[15] S.W. Kim, W. Wang, T.H. Kwon, M.A. Knepper, J. Frokiaer, S. Nielsen, Increased expression of ENaC subunits and increased apical targeting of AQP2 in the kidneys of spontaneously hypertensive rats, Am. J. Physiol. Ren. Physiol. 289 (5) (2005 Nov) F957-F968.

[16] J. Lee, S. Kim, J. Kim, M.H. Jeong, Y. Oh, K.C. Choi, Increased expression of renal aquaporin water channels in spontaneously hypertensive rats, Kidney Blood Press. Res. 29 (1) (2006) 18-23.

[17] J. Lee, Y. Oh, S.W. Kim, Altered renal expression of aquaporin-2 water channels in rats with experimental two-kidney, one clip hypertension, J. Korean Med. Sci. 16 (4) (2001 Aug) 462-466.

[18] S.K. Ma, E.H. Bae, I.J. Kim, C. Choi, J. Lee, S.W. Kim, Altered renal expression of aquaporin water channels and sodium transporters in rats with two-kidney, one-clip hypertension, Kidney Blood Press. Res. 32 (6) (2009) 411-420.

[19] Z.J. Sun, Z.E. Zhang, Historic perspectives and recent advances in major animal models of hypertension, Acta Pharmacol. Sin. 26 (3) (2005 Mar) 295-301.

[20] F. Nobre, C.A. da Silva, E.B. Coelho, H.C. Salgado, R. Fazan Jr, Antihypertensive agents have different ability to modulate arterial pressure and heart rate variability in 2K1C rats, Am. J. Hypertens. 19 (10) (2006 Oct) 1079-1083.

[21] H. Goldblatt, Direct determination of systemic blood pressure and production of hypertension in the rabbit, Proc. Soc. Exp. Biol. Med. 105 (1960 Nov) 213-216.

[22] D.K. Badyal, H. Lata, A.P. Dadhich, Animal models of hypertension and effect of drugs, Indian J. Pharm. 35 (2003) 349-362.

[23] B. Roxas, M. Farjah, R.S. Danziger, Aquaporin-2 transcript is differentially regulated by dietary salt in Sprague-Dawley and Dahl SS/Jr rats, Biochem. Biophys. Res. Commun. 296 (3) (2002 Aug 23) 755-758.

[24] L.M. Burrell, J. Risvanis, R.G. Dean, S.K. Patel, E. Velkoska, C.I. Johnston, Age-dependent regulation of renal vasopressin $\mathrm{V}(1 \mathrm{~A})$ and $\mathrm{V}(2)$ receptors in rats with genetic hypertension: implications for the treatment of hypertension, J. Am. Soc. Hypertens. 7 (1) (2013 Jan) 3-13.

[25] K.J. Livak, T.D. Schmittgen, Analysis of relative gene expression data using real-time quantitative PCR and the 2(-Delta Delta C(T)) method, Methods 25 (4) (2001 Dec) 402-408.
[26] C.A. Davio, G.P. Cricco, R.M. Bergoc, E.S. Rivera, H1 and H2 histamine receptors in N-nitroso-N-methylurea (NMU)-induced carcinomas with atypical coupling to signal transducers, Biochem. Pharmacol. 50 (1) (1995 Jun 29) 91-96.

[27] B. Liu, P.A. Preisig, Compensatory renal hypertrophy is mediated by a cell cycle-dependent mechanism, Kidney Int. 62 (5) (2002) 1650-1658.

[28] U. Hasler, S. Nielsen, E. Feraille, P.Y. Martin, Posttranscriptional control of aquaporin-2 abundance by vasopressin in renal collecting duct principal cells, Am. J. Physiol. Ren. Physiol. 290 (1) (2006 Jan) F177-F187.

[29] J. Lee, D.G. Kang, Y. Kim, Increased expression and shuttling of aquaporin-2 water channels in the kidney in DOCA-salt hypertensive rats, Clin. Exp. Hypertens. 22 (5) (2000 Jul) 531-541.

[30] M. Hiwatari, J.M. Abrahams, T. Saito, C.I. Johnston, Contribution of vasopressin to the maintenance of blood pressure in deoxycorticosterone-salt induced malignant hypertension in spontaneously hypertensive rats, Clin. Sci. (Lond.) 70 (2) (1986 Feb) 191-198.

[31] R.L. Woods, C.I. Johnston, Role of vasopressin in hypertension: studies using the Brattleboro rat, Am. J. Phys. 242 (6) (1982) F727-F732.

[32] R. Lariviere, J. St-Louis, E.L. Schiffrin, Vascular vasopressin receptors in renal hypertensive rats, Am. J. Phys. 255 (4 Pt 2) (1988) H693-H698.

[33] L. Share, J.T. Crofton, Contribution of vasopressin to hypertension, Hypertension 4 (5 Pt 2) (1982) III85-92.

[34] S.W. Kim, Y.S. Jeon, J.U. Lee, D.G. Kang, H. Kook, K.Y. Ahn, et al., Diminished adenylate cyclase activity and aquaporin 2 expression in acute renal failure rats, Kidney Int. 57 (4) (2000 Apr) 1643-1650.

[35] R.R. Campos, E.B. Oliveira-Sales, E.E. Nishi, M.A. Boim, M.S. Dolnikoff, C.T. Bergamaschi, The role of oxidative stress in renovascular hypertension, Clin. Exp. Pharmacol. Physiol. 38 (2) (2011 Feb) 144-152.

[36] E.B. Oliveira-Sales, A.P. Dugaich, B.A. Carillo, N.P. Abreu, M.A. Boim, P.J. Martins, et al., Oxidative stress contributes to renovascular hypertension, Am. J. Hypertens. 21 (1) (2008 Jan) 98-104.

[37] M. Libuy, A. Carreño, Renovascular hypertension, in: R. Rodrigo (Ed.), Advances in Hypertension Research, N: Nova Science Publishers, 2014, pp. 125-151.

[38] M.F. Albertoni Borghese, M.P. Majowicz, M.C. Ortiz, M.F. Delgado, N.B. Sterin Speziale, N.A. Vidal, Renal sodium-glucose cotransporter activity and aquaporin-2 expression in rat kidney during chronic nitric oxide synthase inhibition, Nephron Physiol. 107 (3) (2007) 77-86.

[39] M.F. Albertoni Borghese, L.M. Bettini, C.H. Nitta, F.S. de, M. Majowicz, L.V. Gonzalez Bosc, Aquaporin-2 promoter is synergistically regulated by nitric oxide and nuclear factor of activated T cells, Nephron Extra 1 (1) (2011 Jan) 124-138.

[40] J.S. Handler, H.M. Kwon, Transcriptional regulation by changes in tonicity, Kidney Int. 60 (2) (2001 Aug) 408-411.

[41] F. Montecucco, A. Pende, A. Quercioli, F. Mach, Inflammation in the pathophysiology of essential hypertension, J. Nephrol. 24 (1) (2011 Jan) 23-34.

[42] K. Hocherl, C. Schmidt, B. Kurt, M. Bucher, Inhibition of NF-kappaB ameliorates sepsis-induced downregulation of aquaporin-2/V2 receptor expression and acute renal failure in vivo, Am. J. Physiol. Ren. Physiol. 298 (1) (2010 Jan) F196-F204.

[43] Q. Lin, Y. Genq, S. Lin, Z. Tian, Sirtuin 1 (SIRT1) regulates tumor necrosis factor- $\alpha$ (TNF- $\alpha$-induced) Aquaporin-2 (AQP2) expression in renal medullary collecting duct cells through inhibiting the NFkB pathway, Med. Sci. Monit. Basic Res. 22 (2016) 165-174. 